\title{
Supportive psychotherapy and defense mechanisms: A Comment on the case of Matilde
}

\author{
Jonathan Petraglia ${ }^{1}$
}

The "Case of Matilde" provides us with an important example of how the in-depth study of a single psychotherapy case can shed light on concepts that lie at the very heart of the psychotherapy debate. The therapeutic relationship, patient defense mechanisms, and therapeutic technique are foundational elements of psychodynamic psychotherapy, understanding their interaction will help guide research and practice.

Although the case study enjoyed a central role in the early days of psychotherapy research, randomized controlled trials (RCTs) have replaced case studies as the "gold standard" of empirical psychotherapy research. Nonetheless, Di Riso, Colli, Chessa, Marogna, Condino, et al. (2011) offer a comprehensive analysis that allows readers to get a true sense of what transpires at the clinical level, without ignoring the need for reliable measurement of psychotherapeutic phenomenon.

The first issue of clinical relevance in this case is whether or not Matilde actually received strictly "supportive psychotherapy" during her treatment.

\footnotetext{
${ }^{1}$ Department of Counseling Psychology, McGill University, Canada E-mail: jonathan.petraglia@mail.mcgill.ca
} 
Although the authors, using a Log-linear model, showed that supportive interventions were used more frequently in the first two phases of treatment, there is a spike in expressive interventions during the two middle phase of the therapy, questioning to what degree the treatment was strictly "supportive." Although some variability can be expected during the middle phase of therapy, the fact that the therapist used more interpretive interventions than supportive ones suggests that this may have been a "mixed" supportive/expressive treatment in the way Luborsky (1984) suggests. In fact, when there was a serious threat to the therapeutic alliance in middle phase of treatment ( $3 \& \mathrm{t} 4$ ), the therapist opted to make more interpretations not less as would be expected from established supportive acumen.

Whether or not this new focus in the treatment was actually responsible for resolving the breakdown in the therapy is not clear from the case, but changes during the two middle phases may be either due to, or the result of this increase in interpretive interventions by the therapist or rather a correct "balance" of interpretative and supportive techniques when fundamental conflicts emerged in the middle of psychotherapy. The idea of balancing therapeutic interventions will be explored in greater detail in the next section of this article.

Winston, Winston, Samstag, and Muran (1993) suggest that sustained interpretation is necessary for defense mechanisms to change toward a more adaptive level of functioning in psychodynamic psychotherapy. However, in the case of Matilde we see that therapeutic interventions whose primary aim is not to uncover but rather to support, may play an important, and often overlooked role in the structure and change of a patient's defensive functioning. For example, supporting a patient's defenses is purported to strengthen them thereby increasing their use. However in this case, we see the exact opposite, namely defenses decrease at the end of therapy which is hard to make sense of theoretically and could potentially be due to several causes. 
First, it is possible that the decrease in defense mechanisms witnessed in the case of Matilde is not actually due to the treatment and thus is a product of some other extra-therapeutic event or is the result of measurement error. Since only one DMRS rater was used in the study, it may possibly be the result of inconsistencies in the manner in which the psychotherapy transcripts were rated or rater bias.

Second, it is possible that, as mentioned above, the therapy was actually less "supportive" and more "expressive" than one would expect. As a result, defensiveness changed as expected through the course of therapy. Further evidence for this premise can be found in the fact that Matilde no longer needed to use a "consistent pattern of defenses" (p. 24) by the final timeframe of the treatment. However, and more problematic, is that mature defenses also decreased during this period. It is not clear why this would be the case. Although research has shown that patients who undergo psychodynamic treatment tend to employ less defenses overall (Perry \& Henry, 2004), this does not apply to the mature category of defense mechanisms. In fact, mature defenses typically increase with successful treatment (Hersoug, Bogwald, \& Høglend, 2005). This finding was also supported when a heterogeneous group of treatment was examined in psychiatric practice (Perry \& Henry, 2004). Thus, the case leaves clinicians wondering what exactly happened that would explain this decrease in mature defenses seeing as the defense of self-observation was common at the onset of treatment and that overall, the patient seems to have experienced a positive outcome.

Finally, it is also conceivable that the decrease in defensiveness observed in the case of Matilde was the result of a resolution in central conflicts that signaled the need for the patient to employ a defense. If the therapeutic dyad was working on separation during the final timeframe, this defensive presentation could signal that Matilde is no longer bringing dynamically relevant material to the sessions. This is furthered corroborated by the fact that, as the authors point out "at the end of treatment, defensive 
mechanisms are usually activated" (pp. 24-25), which did not occur with Matilde.

\section{Using supportive interventions with defense mechanisms: What should clinicians do?}

While usually not considered to be as important or curative as the interpretation, supportive techniques make up a large part of what dynamically oriented therapists do in session. They differ from interpretations in that supportive interventions do not confront or make mention of unconscious material. Instead, they aim to support clients' behaviour and generate practical solutions to problems. McWilliams (1994) proposes that when using supportive techniques the therapist interprets feelings and life stressors as opposed to interpreting defenses. McWiliams also indicates that this is especially true for patients who are more disturbed. This may, for example, require the therapist to have the patience to sit and listen to the patient's frustrations or tirades without jumping in to interpret defenses that arise during this process. This probably was not a major concern with Matilde since she did not employ a great deal of immature defenses in therapy. Additionally, supportive techniques such as these sometimes require the therapist to "collude" with the patient's distortions and resistances; however, it does not mean that the therapist agrees with patient's understanding of events.

In the case of Matilde, this would be mean that the therapist did not interfere with her intellectualized understanding of problems and would typically avoid making comments that highlight the lack of emotional meaning in Matilde's descriptions. When Matilde used immature defenses, she typically would rely on disavowal defenses like denial, rationalization, and projection. This presents a more challenging task for the therapist who must both side with the defense without reinforcing its use, as immature defenses are not usually associated with adaptive functioning in life. The therapist must strike a balance between supporting the use of the defense 
while still pointing out to Matilde that she is engaging in a "process" to deal with her intrapsychic conflict.

The idea of combining supportive and interpretive interventions has been studied empirically by Despland, de Roten, Despars, Stigler, and Perry (2001). They proposed that “at each level of a patients' defensive functioning there appears to be some specific range of more exploratory (interpretative) interventions that will be optimal to facilitate growth of the alliance" (p. 162). Although they stated that support alone was not enough in psychotherapy to form a strong alliance, the correct mixture of support and interpretation by therapists was considered necessary for an optimal therapeutic alliance. Despite the fact that Despland and colleagues were interested mostly in alliance, due to the strong link between alliance and outcome in psychotherapy research (Horvath \& Symonds, 1991; Martin, Garske, \& Davis, 2000), it is clear these findings also have implications for outcome as well. In that study, 12 patients seen in ultra-brief (four sessions) were assessed for alliance and defenses. Therapist interventions were also examined and then placed on a continuum from supportive to expressive (ESIL), with expressive techniques considered to be more interpretative than supportive techniques. Thus, the group used this notion to calculate a ratio between the average technique level (supportive versus expressive) and defense level, which was based on maturity level. The results indicated that adjustment scores in-session one predicted alliance scores at session three and four. This result was independent of differing defense scores initially. That is, patients who started off with lower defense scores were still able to form strong alliances when they were well adjusted.

Siefert, Hilsenroth, Weinberger, Blagys, and Ackerman (2006) echoed the sentiment of the Despland group years later when they also concluded that therapists did in fact adjust their supportive and interpretative techniques to patients' defenses early on in Short-term Psychodynamic Psychotherapy (STPP). Siefert and colleagues found that overall defensive functioning predicted the use of both cognitive behavioral and psychodynamic interventions indicating that therapists are using patients' defenses as a 
guiding principle in this form of psychotherapy. However, they were not able to reproduce the results of Despland and colleagues (2001) with respect to defensive functioning and therapeutic alliance. Furthermore, Hersoug, Sexton, and Høglend (2002) confirmed this finding when they found that initial defensive functioning did not predict either alliance or outcome on its own.

In another study, Hersoug, Høglend, and Bogwald (2004) questioned the earlier notion by Despland and colleagues (2001), which assumed that therapist supportive and expressive interventions could be placed on a continuum and then compared to the defensive hierarchy. They concluded that what was assumed to be a "poor" adjustment ratio was correlated with a stronger alliance score in some cases. They also found that when support strategies were given to patients with more adaptive defense scores alliance tended to improve also. This is counterintuitive when we consider that support strategies match with the lower end of the defense continuum to form a more "well adjusted" dyad.

In the case of Matilde, this would mean that she would fall into a "poorly adjusted" category since she receives mostly supportive strategies but her defensive functioning is more in the mid-range. Hersoug and colleagues (2004) explain these discrepancies by suggesting that because Despland and colleagues (2001) studied an ultra-brief form of therapy, it was not necessarily comparable to their naturalistic design, which examined sessions seven and sixteen. None of the above mentioned studies used control groups or experimental manipulation and most used a naturalistic design. All of these factors limit the degree to which these studies can be readily compared because there are a number of factors that could theoretically account for the differences among them.

Hersoug and colleagues (2005), following their previous work, found that interpretations but not support strategies were associated with a decrease in maladaptive defenses over the course of therapy. This relationship was not replicated with respect to adaptive or mid-level defenses. Although adaptive defenses did increase in the sample, neither the use of support nor the use 
of interpretive techniques explained the change. This could help us to understand why Matilde's mature defenses increase at the end of therapy. For example, Drapeau, de Roten, Beretta, Blake, Koerner, and Despland, (2008) studied ultra-brief psychodynamic psychotherapy using sequential analysis, and found that supportive interventions are typically used by therapists to "prepare" patients before making defense interpretations. They also indicated that there are predictable ways in which psychodynamic therapists structure and use therapeutic interventions.

As such, it appears that the relationship between defensive functioning at the beginning of therapy with the therapeutic alliance and outcome is dependent on the therapist's ability to understand and use defenses as part of treatment planning. For example, all of the above mentioned studies did not find a direct relationship between ODF and alliance, only the Despland and colleagues (2001) study found an effect when the concept of adjustment was added. Therefore, it seems that the relationship between defense, alliance, and therapeutic technique is determined at least in part by the therapist's ability to tailor the treatment to patient's characteristics but the role played by supportive interventions is still open for discussion.

Di Riso et al. (2011) have pushed the adjustment debate one step further by calculating the Interaction Adjustment Ratio (IAR), which refers to the ratio of expression level of intervention and the patient's defensive level and is an approach aimed at calculating adjustment in a moment-to-moment fashion in psychotherapy. Most previous studies lack this interactive component thereby ignoring the negotiation that transpires at the human level. An important future step would be to expand this methodology beyond a single case. In doing so, researchers could potentially develop a useful marker for identifying alliance ruptures in psychotherapy.

The authors work highlights the need to quantifiably capture the moment-to-moment aspects of therapeutic adjustment. This process must go beyond simply lining up one averaged variable with another over the course of entire treatments. Research by Petraglia, Perry, Janzen, and Olsen (2009) has also adopted a similar approach for measuring the accuracy of 
defense interpretations. They found that when therapists interpreted patients' defenses in-session from the same DMRS level or higher, that it was associated with a significant increase in the maturity of the defenses used by the patient immediately following the interpretation. Work is currently underway to expand upon these results as only six cases of openended psychodynamic psychotherapy were used in that analysis.

Overall, Di Riso et al. (2011) show that the in-depth quantitative study of a single case is a valid and useful avenue of study for psychotherapy researchers. The strength lies in the considerable amount of data that was collected by the authors to give the reader a true sense of how multiple variables of interest change and interact over the course of treatment.

\section{References}

Despland, J. N., de Roten, Y., Despars, J., Stigler, M., \& Perry, J. C. (2001). Contribution of patient defense mechanisms and therapist interventions to the development of early therapeutic alliance in a brief psychodynamic investigation. The Journal of Psychotherapy Practice and Research, 10, 155-164.

Di Riso, D., Colli, A., Chessa, D., Marogna, C., Condino, V., . . . Mannarini, S. (2011). A supportive approach in psychodynamic-oriented psychotherapy: An empirically supported single case study. Research in Psychotherapy: Psychopathology, Process and Outcome, 14(1), 49-89.

Drapeau, M., de Roten, Y., Beretta, V., Blake, E., Koerner, A., \& Despland, J. N. (2008). Therapist technique and patient defensive functioning in ultra-brief psychodynamic psychotherapy: A LAG sequential analysis. Clinical Psychology and Psychotherapy, 15, 247-255.

Hersoug, A. G., Bogwald, K. P., \& Høglend, P. (2005). Changes of defensive functioning: Does interpretation predict change? Clinical Psychology and Psychotherapy, 12, 288-296.

Hersoug, A. G., Høglend, P., \& Bogwald, K. P. (2004). Is there an optimal adjustment of interpretation to the clients' level of defensive functioning. American Journal of Psychotherapy, 58, 349-361. 
Hersoug, A. G., Sexton, H., \& Høglend, P. (2002). Contribution of defensive functioning to the quality of working alliance and psychotherapy outcome. American Journal of Psychotherapy, 56, 539-554.

Horvath, A. O., \& Symonds, B. D. (1991). Relation between working alliance and outcome in psychotherapy: A meta-analysis. Journal of Counseling Psychology, 38, 139-149.

Luborsky, L. (1984). Principles of psychoanalytic psychotherapy: A manual for supportive-expressive treatment. New York: Basic.

Martin, D. J., Garske, J. P., \& Davis, K. (2000). Relation of the therapeutic alliance with outcome and other variables: A meta-analytic review. Journal of Consulting and Clinical Psychology, 68, 438-450.

McWilliams, N. (1994). Psychoanalytic diagnosis: Understanding personality structure in the clinical process. New York: Guilford Press.

Perry, J. C., \& Henry, M. (2004). Studying defense mechanisms in psychotherapy using the Defense Mechanism Rating Scales. In U. Hentschel, G. Smith, J. G. Draguns \& W. Ehlers (Eds.), Advances in Psychology, 136: Defense mechanisms: Theoretical, research and clinical perspectives (pp. 165-192). New York: Elsevier.

Petraglia, J., Janzen, J. I., Perry, J. C., \& Olson, T. R. (2009). The relationship between therapist interpretation accuracy and in-session change in patients' defensive functioning. Paper presented at the Canadian chapter of the Society for Psychotherapy Research (SPR), Montreal, Canada.

Siefert, C. J., Hilsenroth, M. J., Weinberger, J., Blagys, M. D., \& Ackerman, S. J. (2006). The relationship of client functioning and alliance with therapist technique during short-term psychodynamic psychotherapy. Clinical Psychology and Psychotherapy, 13, 20-33.

Winston, B., Winston, A., Samstag, L. W., \& Muran, J. C. (1993). Patient defense/therapist interventions. Psychotherapy, 31, 478-491. 